\title{
NICE and antibiotic prophylaxis to prevent endocarditis
}

\author{
M. H. Thornhill, ${ }^{* 1,2}$ P. B. Lockhart, ${ }^{3}$ B. Prendergast, ${ }_{1}^{4}$ J. B. Chambers ${ }^{4,5}$ \\ and D. Shanson ${ }^{6}$
}
- Describes new data on the value of antibiotic prophylaxis in preventing infective endocarditis.
- Reviews the development of antibiotic prophylaxis guidelines around the world
- Highlights the current public consultation on the review of the NICE guidelines.
- Encourages dentists to draw their own conclusions about the value of antibiotic prophylaxis, express their views and respond to the consultation.

Infective endocarditis is a devastating disease with high morbidity and mortality. The link to oral bacteria has been known for many decades and has caused on going concern for dentists, patients and cardiologists. Good oral hygiene has long been advocated to prevent endocarditis. Before 2008, antibiotic prophylaxis before invasive dental procedures was also an important strategy for preventing infective endocarditis for patients at risk of the disease in the UK, and still is in most other countries of the world. In 2008, however, NICE published new guidance recommending that antibiotic prophylaxis in the UK should cease. At the time this was a highly controversial decision. New data suggests that there has been a significant increase in the incidence of infective endocarditis since the 2008 guidelines. The 2008 guidance is being reviewed and draft new guidance is being put out for public consultation. This article discusses the issues raised by the new data and the questions that should be addressed in the review and public consultation.

The idea that invasive dental procedures could cause infective endocarditis was first suggested in $1923 .{ }^{1}$ With the advent of antibiotics, the idea developed that antibiotics could be used just before an invasive dental procedure to reduce the risk of endocarditis in susceptible individuals. Guideline committees were soon advising on the antibiotic regimens that should be used and the individuals who should receive cover. Prior to March 2008, all patients at risk of endocarditis were given antibiotic prophylaxis, usually a single $3 \mathrm{~g}$ oral dose of amoxicillin or $600 \mathrm{mg}$ dose of clindamycin, one hour before an invasive dental procedure. But there are longstanding concerns about the efficacy of antibiotic prophylaxis and the risk of adverse drug reactions to the antibiotics used. As a result, the British Society for Antimicrobial Chemotherapy issued new guidance restricting antibiotic use to patients at highest risk of endocarditis. This provoked

\footnotetext{
'Professor of Translational Research in Dentistry, The School of Clinical Dentistry, University of Sheffield; ${ }^{2}$ Adjunct Professor of Oral Medicine, ${ }^{3}$ Professor of Oral Medicine, Department of Oral Medicine, Carolinas Medical Centre, Charlotte, North Carolina, USA; ${ }^{4}$ Consultant Cardiologist, ${ }^{5}$ Professor of Clinical Cardiology, Guy's and St Thomas' Hospitals, London; ${ }^{6}$ Honourary Consultant Microbiologist at Great Ormond Street Hospital for Children, London

*Correspondence to: Professor Martin Thornhill Email: M.Thornhill@Sheffield.ac.uk

Tel: +44 1142717857
}

\section{Refereed Paper}

Accepted 13 May 2015

DOI: 10.1038/sj.bdj.2015.496

${ }^{\circledR}$ British Dental Journal 2015; 218: 619-621 outrage on the part of many, who felt this was a step too far in reducing the use of antibiotic prophylaxis and prompted NICE to carry out a review. They found little if any evidence to show antibiotic prophylaxis was of benefit, although they did not consider certain types of study, including animal studies, in the review. No observational or case controlled studies were available to determine antibiotic prophylaxis efficacy and most crucially, no randomised controlled trial has ever been performed to provide definitive evidence. NICE were also concerned that the number and severity of adverse drug reactions caused by antibiotic prophylaxis would outweigh the benefit from any cases of infective endocarditis that would be prevented. On this basis they also concluded that antibiotic prophylaxis was not cost effective. Consequently, the NICE guidance published in March 2008 recommended that antibiotic prophylaxis before invasive dental procedures should stop. ${ }^{2}$

Dentists responded well to this recommendation and levels of prophylaxis prescribing dropped dramatically. However, most cardiologists and many dentists remained concerned that patients at the highest risk of endocarditis, such as those with prosthetic heart valves or a previous history of infective endocarditis, should continue to receive antibiotic cover. ${ }^{3}$ So, although antibiotic prophylaxis prescribing fell by nearly $90 \%$ in the five years after the NICE guidelines, around 15,000 prescriptions a year are still being issued ${ }^{4}$ - mainly targeting individuals at highest risk. ${ }^{3}$ Some of this may be for other purposes but it is clear that many dentists feel pressured, or that it is their responsibility, to prescribe antibiotic prophylaxis for some of their patients. ${ }^{3}$ This pressure comes from patients and their cardiologists ${ }^{3}$ and is supported by the fact that other guideline committees, including those in mainland Europe ${ }^{5}$ and America ${ }^{6}$, drew a different conclusion to NICE when they reviewed the evidence. They felt that the risks associated with endocarditis in the highest risk groups (those more likely to have a bad outcome if they developed endocarditis as well as those more likely to develop it) were likely to exceed the risks associated with adverse drug reactions. So in the absence of evidence either for or against antibiotic prophylaxis, they concluded that it was safer to recommend it for high-risk groups. As a result, the UK is now the only place that does not recommend antibiotic prophylaxis for high-risk individuals. For many dental practitioners this has been a cause for concern.

There is little doubt that bacteria originating from the mouth account for 35-45\% of cases of infective endocarditis. The question is, how do bacteria get from the mouth into the circulation to infect the heart valves of susceptible individuals? Although invasive dental procedures are one possibility, there is good evidence that bacteria can enter the circulation as a result of daily activities such as tooth brushing, flossing and chewing food, particularly in those with poor oral hygiene and periodontal disease. ${ }^{7,8}$ Some 
have argued that the frequency of bacteraemia resulting from daily activities is so much greater than that from invasive dental procedures, that there is no point in giving antibiotic prophylaxis - but really, we do not know. Intuitively, most dentists feel that really good oral hygiene is likely to play an important role in protecting those at risk of infective endocarditis from developing the disease. However, they also worry about how best to achieve good oral hygiene in these individuals and worry about the possibility that oral hygiene procedures themselves, particularly scaling, might put them at risk. So despite the NICE guidelines, there is ongoing worry and concern.

Guideline committees would really like to see a randomised controlled trial of antibiotic prophylaxis to prove that it is, or is not, effective (although several such trials producing the same result would be needed to provide certainty). The problem is that hundreds of patients need to be given antibiotic prophylaxis to prevent one case of endocarditis. ${ }^{4}$ Furthermore, infective endocarditis is a relatively uncommon condition. This means any study would need to include hundreds of thousands of high-risk individuals randomised to placebo or active drug and followed for five years or more to attain sufficient statistical power. Such a study came close to being funded but the cost and complexity of this type of study is huge and funders have felt unable to justify such enormous expenditure on an uncommon condition when treatment trials of conditions such as cancer, diabetes etc would have similar or greater impact but much lower cost. Big ethical issues also face a randomised controlled trial of antibiotic prophylaxis on higher risk individuals, particularly in countries where antibiotic prophylaxis is the standard of care. In reality there is little prospect of getting data from a randomised controlled trial in the foreseeable future.

In the absence of a randomised controlled trial, an observational study comparing the incidence of infective endocarditis in a population who received antibiotic prophylaxis and another that did not would provide the next best level of evidence. Introduction of the NICE guidelines in March 2008 made such a study possible. By comparing the incidence of infective endocarditis before and after March 2008 we can get some idea of the effect, if any, of antibiotic prophylaxis. In March this year, some of the authors of this opinion piece, published a paper in the Lancet ${ }^{4}$ that did exactly this. What we found was a very significant $88 \%$ fall in antibiotic prophylaxis prescribing in the five years following the NICE guidelines and a highly significant increase in the incidence of infective endocarditis above what would have been expected from projection of the pre-NICE trend. The data suggested that by March 2013 there had been 419 more infective endocarditis cases a year than expected. The 95\% confidence limits (CI) suggest this figure could be as high as 743 or as low as 95 extra cases. Although the study did not identify a significant increase in mortality, the overall immediate mortality of infective endocarditis patients in the study was $15.7 \%$ during this period, suggesting that the extra cases could have resulted in 66 extra deaths per year (95\% CI: 15-117). This data also suggest that 277 prescriptions of antibiotic prophylaxis are required to prevent one case of infective endocarditis. The problem with observational data is that just because we see a relationship; it does not prove that the fall in antibiotic prophylaxis caused the increase in infective endocarditis. However, a careful search for other possible causes of the increase in incidence was unable to identify any satisfactory alternative explanations. Furthermore, a statistical method called 'change point analysis' confirmed a very close time relationship between the fall in antibiotic prophylaxis prescribing and the increase in incidence of infective endocarditis that is otherwise hard to explain.

Another paper from our group, recently published in the Journal of Antimicrobial Chemotherapy, ${ }^{9}$ looked at yellow card reporting of adverse drug reactions to amoxicillin and clindamycin prescribed as single oral dose antibiotic prophylaxis. There were no recorded cases of fatal reaction with amoxicillin prophylaxis and only two reactions each year sufficiently serious to warrant reporting. This confirmed the findings of a previous study that over a 35-year period identified not a single fatal adverse reaction report following use of a $3 \mathrm{~g}$ oral amoxicillin sachet. ${ }^{10}$ For those not allergic to penicillin, therefore, amoxicillin prophylaxis would appear to be very safe - and much safer than previously thought. In contrast, for clindamycin there were four non-fatal adverse reactions reported each year and one fatal reaction every three years. The majority of clindamycin adverse reactions were Clostridium difficile infections. Considering the much lower level of clindamycin antibiotic prophylaxis prescribing this was a much higher rate of reactions than expected suggesting that a different alternative to amoxicillin needs to be identified.

While by no means conclusive, this new evidence suggests that antibiotic prophylaxis may prevent a significant number of cases of infective endocarditis and, at least for those without a history of penicillin allergy, that amoxicillin antibiotic prophylaxis is very safe with a low likelihood of adverse reaction. Hard data like this make it possible to perform a health economic analysis of the cost effectiveness of antibiotic prophylaxis, where real figures can be substituted for many of the assumptions and estimates that bedevilled earlier analyses. If the recent data are correct, then amoxicillin antibiotic prophylaxis proves cost effective, particularly if it is restricted to those individuals most at risk of developing infective endocarditis.

NICE were made aware of the data to be published in the Lancet in November 2014 and promptly announced a review of their guidance. This has been completed and draft new guidance will be made available on their website (https://www. nice.org.uk/guidance/indevelopment/gidcgwave0748) for public consultation. The consultation period is very short -1 to 29 June 2015 - and we urge you to respond to the consultation to express your views and concerns, whatever they may be. The BDA is registered with NICE as a stakeholder and it is to be hoped that you will also inform them of your views and that they will also respond to the consultation.

The information available at the time this opinion piece was written suggests that NICE has decided that there is insufficient evidence on which to change the guidelines because the new data are not from a randomised controlled trial and do not prove that the fall in antibiotic prophylaxis caused the increase in infective endocarditis. This is an interesting stance since NICE changed the guidance in 2008 without having strong evidence one way or the other and certainly not the level of evidence now available.

Although NICE produced a list of conditions that put patients at higher risk of infective endocarditis in 2008, they did not say what more should be done to reduce the risk of endocarditis in these individuals. It seems to imply that these individuals might benefit from antibiotic prophylaxis yet NICE provide no clear guidance about when or if any individuals should be exempted from the no antibiotic prophylaxis guidance. The risk posed by poor oral hygiene and invasive dental procedures in high-risk individuals causes significant concern for many dentists, patients and their cardiologists. Clearer guidance on which patients should be exempted from the NICE guidance would benefit everyone - particularly when ongoing prescribing levels suggest that many patients are being exempted every year by their physicians and dentists.

There is good evidence that a transient bacteraemia is likely to occur when high-risk 
patients with poor oral hygiene and periodontal disease undergo invasive dental procedures. ${ }^{7,8,11,12}$ Even if antibiotic prophylaxis is deemed to be of no benefit, should dentists advise such patients of the signs of infective endocarditis and what action to take to ensure rapid treatment and a favourable outcome? The British Heart Foundation produces such a warning card, which is not currently promoted to dentists. Should the current NICE review consider whether dentists should provide such advice to their high-risk patients when they undergo invasive procedures?

In 2008, NICE also mentioned the importance of maintaining good oral health. However, dentists and their patients need clearer guidance how this should be achieved. What advice should cardiologists and dentists be giving their patients? More importantly, what are the goals of treatment and how should they be achieved? How intensive should periodontal therapies be and should higher risk individuals receive antibiotic cover for these, at least until their oral hygiene has improved to a sufficiently good level? What should be the timing and frequency of both acute and maintenance treatment? And, if, like most dentists, NICE really thinks maintenance of good oral hygiene plays an important role in preventing infective endocarditis, should it not recommend free dental care for the relatively low number of individuals at high-risk of endocarditis - just as pregnant women get free NHS dental care? It can only be hoped that the 2015 review will consider these questions and provide some answers.

It will be a concern should NICE decide that there is insufficient evidence to revise its guidance because the new data are not from a randomised controlled trial. NICE guidance should be reviewed every two years and even guidelines on the static list are supposed to undergo high-level review every five years. Unusually, the guidance on antibiotic prophylaxis for infective endocarditis was put on the static list in 2008 where it has remained unreviewed for more than seven years. So NICE should undertake a thorough review now (at the very least) and consider the issues above where patients and clinicians require clearer guidance. Furthermore, like the other guideline committees around the world, they should consider both the risk and benefits of their recommendation while waiting for the definitive answer provided by a randomised controlled trial.

Although we cannot be certain that the Lancet data are correct, it is the best we have while we await a randomised controlled trial. If such a trial ultimately demonstrates that the Lancet data is correct, then the recommendation not to give antibiotic prophylaxis in the interim could result in an extra 419 cases of endocarditis a year (95\% CI: 95-743) - including a possible extra 66 deaths $(95 \%$ CI: 15-117).

If, on the other hand, a randomised controlled trial ultimately proves the Lancet data wrong, a recommendation to give antibiotic prophylaxis in the interim could, according to the Journal of Antimicrobial Chemotherapy data, result in zero deaths and two adverse drug reactions severe enough to be reported a year. This would be higher if clindamycin were also recommended. However, one would expect NICE to recommend a different antibiotic (perhaps erythromycin ${ }^{13}$ ) or no antibiotic prophylaxis for those allergic to penicillin.

This risk assessment suggests that it would be much safer to recommend antibiotic prophylaxis than no antibiotic prophylaxis while waiting on randomised controlled trial data. This would be even more the case if prophylaxis were restricted to those at high-risk.

This observation is important but does not mean that antibiotic prophylaxis is more useful than maintenance of good oral hygiene in preventing infective endocarditis. Both may well play a role. It seems very likely that regular small bacteraemias from daily activities pose a significant threat to patients at risk of endocarditis. But that does not mean that occasional large bacteraemias from invasive dental procedures are no threat. Our aim should be to minimise all causes of bacteraemia in susceptible individuals.

It is to be hoped that the current NICE review will address these issues that caused so much concern for dentists, patients and cardiologists in 2008 and continue to do so in the light of the newly emerging data.
Ultimately, we all want to do what is best for our patients. We therefore urge dentists who have an opinion on this issue to respond to the NICE public consultation, whatever their view.

1. Lewis T, Grant R. Observations relating to subacute infective endocarditis. Heart 1923; 10: 21-77.

2. National Institute for Health and Clinical Excellence Prophylaxis against infective endocarditis. 2008. Online information available at https://www.nice. org.uk/guidance/cg64 (accessed March 2015).

3. Dayer M J, Chambers J B, Prendergast B, Sandoe J A Thornhill M H. NICE guidance on antibiotic prophylaxis to prevent infective endocarditis: a survey of clinicians) attitudes. OJM 2013; 106: 237-243.

4. Dayer M J, Jones S, Prendergast B, Baddour L M, Lockhart P B, Thornhill M H. Incidence of infective endocarditis in England, 2000-13: a secular trend, interrupted time-series analysis. Lancet 2015; 385: 1219-1228.

5. Habib G, Hoen B, Tornos P et al. Guidelines on the prevention, diagnosis, and treatment of infective endocarditis (new version 2009): the task force on the prevention, diagnosis, and treatment of infective endocarditis of the European Society of Cardiology (ESC). Endorsed by the European Society of Clinical Microbiology and Infectious Diseases (ESCMID) and the International Society of Chemotherapy (ISC) for Infection and Cancer. Eur Heart J 2009; 30: 2369-2413.

6. Wilson W, Taubert K A, Gewitz M et al. Prevention of infective endocarditis: guidelines from the American Heart Association: a guideline from the American Heart Association Rheumatic Fever, Endocarditis, and Kawasaki Disease Committee, Council on Cardiovascular Disease in the Young, and the Council on Clinical Cardiology, Council on Cardiovascular Surgery and Anesthesia, and the Quality of Care and Outcomes Research Interdisciplinary Working Group. Circulation 2007; 116: 1736-1754.

7. Lockhart P B, Brennan M T, Sasser H C, Fox P C, Paster B J, Bahrani-Mougeot F K. Bacteremia associated with toothbrushing and dental extraction. Circulation 2008; 117: 3118-3125.

8. Lockhart P B, Brennan M T, Thornhill M et al. Poor oral hygiene as a risk factor for infective endocarditis-related bacteremia. J Am Dent Assoc 2009; 140: 1238-1244.

9. Thornhill M H, Dayer M J, Prendergast B, Baddour $L M_{1}$ Jones $S$, Lockhart $P$ B. Incidence and nature of adverse reactions to antibiotics used as endocarditis prophylaxis. J Antimicrob Chemother 2015 [Epub ahead of print].

10. Lee P, Shanson D. Results of a UK survey of fatal anaphylaxis after oral amoxicillin. J Antimicrob Chemother 2007; 60: 1172-1173.

11. Bahrani-Mougeot F K, Paster B J, Coleman S, Ashar J, Barbuto S, Lockhart P B. Diverse and novel oral bacterial species in blood following dental procedures. J Clin Microbiol 2008; 46: 2129-232.

12. Lockhart P B. An analysis of bacteremias during dental extractions. A double-blind, placebo-controlled study of chlorhexidine. Arch Intern Med 1996; 156: 513-520.

13. Shanson D C, Akash S, Harris M, Tadayon M. Erythromycin stearate, $1.5 \mathrm{~g}$, for the oral prophylaxis of streptococcal bacteraemia in patients undergoing dental extraction: efficacy and tolerance. J Antimicrob Chemother 1985; 15: 83-90. 